\title{
THE STRESSES AROUND A FAULT OR CRACK IN DISSIMILAR MEDIA
}

\author{
By M. L. Williams
}

\begin{abstract}
In order to investigate some problems of geophysical interest, the usual consideration of symmetrical or antisymmetrical loading of an isotropic homogeneous plate containing a crack was extended to the case where the alignment of the crack separates two separate isotropic homogeneous regions. It develops that the modulus of the singular behavior of the stress remains proportional to the inverse square root of the distance from the point of the crack, but the stresses possess a sharp oscillatory character of the type $r^{-\frac{1}{2}} \sin (b \log r)$, which seems to be confined quite close to the point, as well as a shear stress along the material joint line as long as the materials are different.

The off-fault areas of high strain energy release reported by St. Amand for the White Wolf fault are qualitatively shown to be expected.
\end{abstract}

As a logical extension of a previous plane-stress or plane-strain problem ${ }^{1}$ which dealt with the stress distribution at the base of a stationary crack in an isotropic homogeneous material, it is proposed to discuss the characteristic behavior of the stress in the vicinity of a crack between the plane-bounding surfaces of two dissimilar materials. The problem was suggested by a possible application to situations in geological investigations dealing with fault lines along the interface between two layers of rock strata, but it may also apply to certain weld joints which, owing to faulty joining techniques, or for that matter applied loading, develop cracks along the original weld line.

The geometry considered is that of a material $M_{1}$ occupying the upper half plane and a material $M_{2}$ in the lower half plane, joined without residual stress along the positive $x$-axis, or positive radial direction $r$ measured from the origin along $\psi=0$. The elastic plane stress solution is therefore desired for unloaded edges along the negative $x$-axis.

In the previous homogeneous case where $M_{1}=M_{2}$ it was found that the stresses near the base of the crack became (mathematically) infinite according to an inverse square-root law, $\sigma \sim r^{-\frac{1}{2}}$. The interesting question which arises concerns how the character of the stress is changed as a result of the discontinuity of the material properties across the line of erack prolongation.

\section{OUtLine of Solution}

Reviewing the method of solution which is naturally very similar to the homogeneous case, a biharmonic stress function $\chi(r, \psi)$, that is, a solution of

$$
\nabla^{4} \chi(r, \psi)=0,
$$

is to be found such that the normal stress, $\sigma_{\psi}$, and shear stress, $\tau_{r \psi}$ vanish along $\psi= \pm \pi$, and further that the displacements and stresses are continuous across the material demarcation line $\psi=0$.

\footnotetext{
Manuseript received for publication August 8, 1958.

${ }^{1}$ M. L. Williams, "On the Stress Distribution at the Base of a Stationary Crack," Jour. Applied Mechanies, March, 1956.
} 
Typical solutions are chosen of the form

$$
\begin{aligned}
\chi(r, \psi)=r^{\lambda+1} F(\psi)= & r^{\lambda+1}\{a \sin (\lambda+1) \psi+b \cos (\lambda+1) \psi \\
& +c \sin (\lambda-1) \psi+d \cos (\lambda-1) \psi\},
\end{aligned}
$$

where the usual relations between stresses, displacements, and stress function are given $^{2}$ as

$$
\begin{gathered}
\sigma_{r}=\frac{1}{r^{2}} \frac{\partial^{2} \chi}{\partial \psi^{2}}+\frac{1}{r} \frac{\partial \chi}{\partial r}=r^{\lambda-1}\left[F^{\prime \prime}(\psi)+(\lambda+1) F(\psi)\right] \\
\sigma_{\theta}=\frac{\partial^{2} \chi}{\partial r^{2}}=r^{\lambda-1} \lambda(\lambda+1) F(\psi) \\
\tau_{r \psi}=-\frac{1}{r} \frac{\partial^{2} \chi}{\partial r \partial \theta}+\frac{1}{r^{2}} \frac{\partial \chi}{\partial \theta}=-\lambda r^{\lambda-1} F^{\prime \prime}(\psi) \\
u_{\psi}=\frac{1}{2 \mu} r^{\lambda}\left\{-F^{\prime}(\psi)-4(1-\sigma)[c \cos (\lambda-1) \psi-d \sin (\lambda-1) \psi]\right\} \\
u_{r}=\frac{1}{2 \mu} r^{\lambda}\{-(\lambda+1) F(\psi)+4(1-\sigma)[c \sin (\lambda-1) \psi+d \cos (\lambda-1) \psi]\} .
\end{gathered}
$$

Here $\mu$ is the shear modulus and $\sigma$, in terms of Poisson's ratio, $\nu$, is $\sigma \equiv \nu /(1+\nu)$. The primes denote differentiation with respect to $\psi$.

As a matter of notation, let the quantities in the regions $M_{1}$ and $M_{2}$ have the appropriate subscript $F_{1}, F_{2} ; \lambda_{1}, \lambda_{2} ; a_{1}, a_{2} ; \nu_{1}, \nu_{2}$; etc., respectively. With this convention the first four boundary conditions for free edges at $\psi= \pm \pi$ become

$$
F_{1}(\pi)=F_{1}^{\prime}(\pi)=F_{2}(-\pi)=F_{2}^{\prime}(-\pi)=0
$$

and upon noting that $\lambda_{1} \equiv \lambda_{2}$ in order that the second four boundary conditions be independent of $r$, there results

$$
\begin{aligned}
F_{1}(0) & =F_{2}(0) \\
F_{1}^{\prime}(0) & =F_{2}^{\prime}(0) \\
\frac{1}{2 \mu_{1}}\left[-F_{1}^{\prime}(0)-4 c_{1}\left(1-\sigma_{1}\right)\right] & =\frac{1}{2 \mu_{2}}\left[-F_{2}^{\prime}(0)-4 c_{2}\left(1-\sigma_{2}\right)\right] \\
\frac{1}{2 \mu_{1}}\left[-(\lambda+1) F_{1}(0)+4 d_{1}\left(1-\sigma_{1}\right)\right] & =\frac{1}{2 \mu_{2}}\left[-(\lambda+1) F_{2}(0)+4 d_{2}\left(1-\sigma_{2}\right)\right] .
\end{aligned}
$$

2. Timoshenko and J. N. Goodier, Theory of Elasticity (New York: McGraw-Hill, 1951). 
Substitution of $F_{1}(\psi)$ and $F_{2}(\psi)$ in (8)-(15) leads to the following eight homogeneous linear equations in the eight unknowns $a_{1}, a_{2} \cdots d_{1}, d_{2}$.

$$
\begin{aligned}
& a_{1} \sin (\lambda+1) \pi+b_{1} \cos (\lambda+1) \pi+c_{1} \sin (\lambda-1) \pi+d_{1} \cos (\lambda-1) \pi=0 \\
& -a_{2} \sin (\lambda+1) \pi+b_{2} \cos (\lambda+1) \pi-c_{2} \sin (\lambda-1) \pi+d_{2} \cos (\lambda-1) \pi=0 \\
& \begin{array}{r}
a_{1}(\lambda+1) \cos (\lambda+1) \pi-b_{1}(\lambda+1) \sin (\lambda+1) \pi+c_{1}(\lambda-1) \cos (\lambda-1) \pi \\
-d_{1}(\lambda-1) \sin (\lambda-1) \pi=0
\end{array} \\
& a_{2}(\lambda+1) \cos (\lambda+1) \pi+b_{2}(\lambda+1) \sin (\lambda+1) \pi+c_{2}(\lambda-1) \cos (\lambda-1) \pi \\
& \quad+d_{2}(\lambda-1) \sin (\lambda-1) \pi=0 \\
& b_{1}+d_{1}=b_{2}+d_{2} \\
& (\lambda+1) a_{1}+(\lambda-1) c_{1}=(\lambda+1) a_{2}+(\lambda-1) c_{2} \\
& 4\left(1-\sigma_{1}\right) c_{1}=4 k\left(1-\sigma_{2}\right) c_{2}+(k-1)\left[(\lambda+1) a_{2}+(\lambda-1) c_{2}\right] \\
& 4\left(1-\sigma_{1}\right) d_{1}=4 k\left(1-\sigma_{2}\right) d_{2}-(k-1)(\lambda+1)\left[b_{2}+d_{2}\right],
\end{aligned}
$$

where the shear modulus ratio $k \equiv \mu_{1} / \mu_{2}$ has been introduced.

A nontrivial solution for the constants exists if the determinant of the eight equations vanish. After some algebraic simplification, the determinant can be written in the form

$$
\cot ^{2} \lambda \pi+\left[\frac{2 k\left(1-\sigma_{2}\right)-2\left(1-\sigma_{1}\right)-(k-1)}{2 k\left(1-\sigma_{2}\right)+2\left(1-\sigma_{1}\right)}\right]^{2}=0
$$

\section{The Homogeneous Case}

When the material in both regions is the same, $\sigma_{1}=\sigma_{2}$ and $k=\mu_{1} / \mu_{2}=1$. Thus (16) reduces to the simple form

$$
\cot ^{2} \lambda \pi=0
$$

and by inspection the eigen values are

$$
\begin{aligned}
\lambda & =(2 n+1) / 2 \quad n=0,1,2, \cdots \\
& =1 / 2,3 / 2,5 / 2, \cdots
\end{aligned}
$$

where negative values of $n$ have been excluded so that the physical displacements $u_{\psi}$ and $u_{r}$ are finite as the origin is approached, i.e., the stress function and its first derivative exist along the boundary.

Thus an infinite number of $\lambda_{n}$ exist with the lowest- the one controlling the stress behavior near the base of the crack, $r \sim 0$-being $\lambda_{\text {min }}=1 / 2$. Hence the local stress behavior, from (3) to (5), is of order $\sigma \sim r^{\lambda-1}=r^{-\frac{1}{2}}$. 


\section{The Bmaterial Case}

It is immediately evident from (16) that no real solutions exist for different materials as this equation is the sum of two positive terms equated to zero, which solution is the homogeneous case. Nothing excludes the admissibility of complex values of the eigen parameter, however, and upon assuming $\lambda \equiv \lambda_{r}+i \lambda_{j}$ it is quickly determined that

$$
\begin{aligned}
& \frac{\left[\tan ^{2} \lambda_{r} \pi+1\right] \tanh \lambda_{3} \pi}{\tan ^{2} \lambda_{r} \pi+\tanh ^{2} \lambda_{j} \pi}= \pm \frac{2 k\left(1-\sigma_{2}\right)-2\left(1-\sigma_{1}\right)-(k-1)}{2 k\left(1-\sigma_{2}\right)+2\left(1-\sigma_{1}\right)} \\
& \frac{\tan \lambda_{r} \pi\left[1-\tanh ^{2} \lambda_{j} \pi\right]}{\tan ^{2} \lambda_{r} \pi+\tanh ^{2} \lambda_{j} \pi}=0 .
\end{aligned}
$$

Two sets of solutions are possible. The first, from (20), if $\tan \lambda_{r} \pi=0$ giving

$$
\begin{aligned}
& \lambda_{r}=n=0,1,2,3, \ldots \\
& \lambda_{j}= \pm \frac{1}{\pi} \operatorname{coth}^{-1}\left[\frac{2 k\left(1-\sigma_{2}\right)-2\left(1-\sigma_{1}\right)-(k-1)}{2 k\left(1-\sigma_{2}\right)+2\left(1-\sigma_{1}\right)}\right] \neq 0
\end{aligned}
$$

The second solution occurs, from (20), if $\tan \lambda_{r} \pi=\infty$ giving

$$
\begin{aligned}
& \lambda_{r}=1 / 2,3 / 2,5 / 2, \cdots \\
& \lambda_{j}= \pm \frac{1}{\pi} \tanh ^{-1}\left[\frac{2 k\left(1-\sigma_{2}\right)-2\left(1-\sigma_{1}\right)-(k-1)}{2 k\left(1-\sigma_{2}\right)+2\left(1-\sigma_{1}\right)}\right]
\end{aligned}
$$

which is observed to approach the homogeneous material solution $\left(\lambda_{j} \rightarrow 0\right.$, as $\sigma_{1} \rightarrow \sigma_{2}$ and $k \rightarrow 1$ ).

\section{Discussion of the Solution}

The stresses from the second solution are therefore seen to behave, for the minimum eigen value, according to

$$
\sigma \sim r^{\lambda-1} \sim r^{\left(\lambda_{r}-1\right) \pm i \lambda_{j}}
$$

or

$$
\sigma \sim r^{-1 / 2}\left(\begin{array}{c}
\sin \\
\cos
\end{array}\right)\left(\lambda_{j} \log r\right)
$$

which is of an oscillating character with its maximum modulus determined by $r^{-\frac{1}{3}}$ which is the same bound as determined in the case of homogeneous materials. Indeed, for this special case it was shown that $\lambda_{j}=0$ for all roots, and the results are seen to be identical with those of the previous more comprehensive analysis. 
Turning to a further consideration of the oscillatory behavior, consider a case when $\nu_{1}=\nu_{2}$. Then

$$
\left|\lambda_{j}\right|=\left|\frac{1}{\pi} \tanh ^{-1} \frac{1-\nu}{2} \cdot \frac{k-1}{k+1}\right| \approx \frac{1}{\pi} \tanh ^{-1} \frac{1-\nu}{2} \sim \frac{1}{5}
$$

so that the first zero as the origin is approached would be of the order of $\left|\lambda_{j} \log r\right| \sim$ $\pi / 2$ or $|\log r| \sim-7$ and hence with the radial dimension expressed in terms of some characteristic distance in the plane of the plate, say $R, r / R \sim e^{-7} \sim 10^{-3}$. A pronounced oscillatory character of the stress, on the basis of this qualitative calculation, would therefore appear to be confined quite close to the base of the crack.

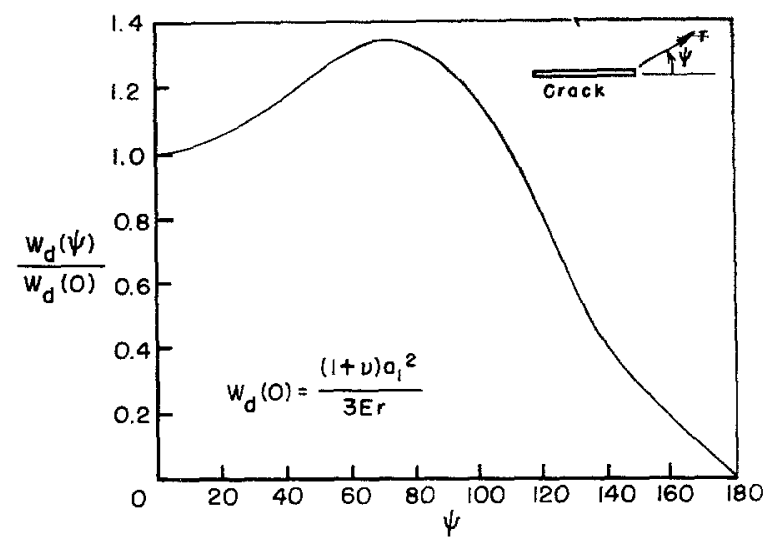

Fig. 1. Distortion energy distribution at constant radius for symmetric fault loading.

The first solution, however, bears further examination owing to the existence of the pure imaginary solution $\lambda=i \lambda_{j}$ for $n=0$. The displacements then appear to be finite $u \sim r^{\lambda} \sim r^{ \pm i \lambda_{i}}$ so that

$$
u \sim\left(\begin{array}{l}
\sin \\
\cos
\end{array}\right)\left(\lambda_{j} \log r\right)
$$

and stresses of the form

$$
\sigma \sim r^{-1}\left(\begin{array}{l}
\sin \\
\cos
\end{array}\right)(\lambda, \log r)
$$

which would result in a stronger stress singularity than the $r^{-\frac{1}{2}}$ behavior found in the second solution.

It is believed, nevertheless, that the proper interpretation would require that the displacements tend to zero as the base of the crack is approached. Thus the value of $n=0$ would be excluded and the two sets of eigen values would be intertwinedthe lowest, however, being generated from the second set. The latter set yields the physically expected homogeneous case as the materials $M_{1}$ and $M_{2}$ become the same.

On this basis, therefore, one would expect very high strain concentrations at the ends of a fault. Indeed, for the elastic homogeneous case it was shown that the strain 
energy stored in the medium was not uniformly distributed over a peripheral region about the end of the crack, but at a constant radius was one-third higher at \pm 70 degrees to the left or right of the direction of crack prolongation than directly ahead (fig. 1). Further graphs and additional formulas for symmetrical and antisymmetrical loading are given in detail for the case of similar materials in Williams, op. cit. (n. 1).

Dr. Beno Gutenberg has pointed out the interesting feature to the writer that the areas of maximum energy release reported by St. Amand ${ }^{3}$ also seems to occur ahead, but to the side, of the fault direction and hence is qualitatively in agreement with the previous calculations.

\footnotetext{
${ }^{3}$ Pierre St. Amand, "Two Proposed Measures of Seismicity," Bull. Seism. Soc. Am., Vol. 46, No. 1, January, 1956.

Calffornia Institute of Technology, Pasadena, California.
} 